J. Austral. Math. Soc. 19 (Series A) (1975), 413-425.

\title{
SOME RESULTS CONCERNING A TRANSFORMATION SEMIGROUP
}

\author{
J. S. V. SYMONS \\ (Received 19 May 1972) \\ Communicated by G. B. Preston
}

\section{Introduction}

The full transformation semigroup $\mathscr{T}(X)$ is extremely important. As far back as 1952, Malcev (1952) showed that its automorphism group consisted of mappings of the form $g^{-1} \cdot g, g \in \mathscr{G}(X)$. The present work is devoted to a generalization of this result.

The semigroup we consider is $\mathscr{T}(X, Y), Y \subseteq X$, comprised of those mappings in $\mathscr{T}(X)$ whose range is contained in $Y$. To the extent that $\mathscr{T}(X, X)=\mathscr{T}(X)$ we may count $\mathscr{T}(X, Y)$ a generalization of $\mathscr{T}(X)$.

We answer two questions:

A What are the automorphism of $\mathscr{T}(X, Y)$ ?

B When are two $\mathscr{T}(X, Y)$ isomorphic?

For $|Y|>2$ the results are as one expects:

A The automorphism of $\mathscr{T}(X, Y)$ are "inner" induced by those permutations of $X$ which are also permutations of $Y$.

B $\mathscr{T}(X, Y)$ is determined by the cardinals $|Y|$ and $|X| Y \mid$. (Not $|X|$ and $|Y|$ : See $§ 5)$.

It is when $|Y|=2$ that the exceptions arise. In $\S 2$ we discuss this case and show that there are many more automorphisms than inner automorphisms. We characterize these "outer" automorphisms by means of class of permutations of $\mathscr{P}(X)^{*}$ with pleasant properties.

In $\$ 1$ we reduce the calculation of Aut $\mathscr{T}(X, Y)$ to determining a simpler group which we denote by $\operatorname{Aut}_{Y} \mathscr{T}(X, Y)$. It turns out that any element of Aut $_{Y} \mathscr{T}(X, Y)$ may also be considered as an automorphism of $\mathscr{T}(X, 2)$, where 2 is any doubleton subset of $Y$; and in $\S 3$ the results of $\$ 2$ are used to determine

* The power set of $X$. 
the general automorphism. Thus the case $|Y|=2$, far from being the low order abberation to which one is accustomed, (c.f. automorphisms and normal subgroups of $\mathscr{G}(X)$. See Scott (1964) Chapter 11), is actually the cornerstone of the theory.

We'discuss some extensions in $\$ 4$. In particular, we exhibit the automorphism group of $\mathscr{S}(X, Y)$, a semigroup introduced by Magill (1966). It is to this end that we prove rather more general results in $\S 1$ than seems warranted by our major intentions.

In $\S 5$ we answer $\mathrm{B}$ by an examination of $\operatorname{Aut}_{Y} \mathscr{T}(X, Y)$. In the exceptional case, $|Y|=2, \mathscr{T}(X, Y)$ is determined by $2^{|X \backslash Y|}$ rather than $|X| Y \mid$.

\section{Preliminaries; basic results}

Our notation is that of Clifford and Preston (1967) with some additions and departures. If $X$ is a set then the full transformation semigroup on $X$ will be denoted by $\mathscr{T}(X)$. Also, if $Y \subseteq X$ then

and

$$
\mathscr{T}(X, Y)=\{\alpha \in \mathscr{T}(X) ; X \alpha \subseteq Y\}
$$

$$
\mathscr{G}(X, Y)=\{g \in \mathscr{G}(X) ; g \mid Y \in \mathscr{G}(Y)\}
$$

where $\mathscr{G}(X)$ is the full permutation group on $X$.

If $S \leqq \mathscr{T}(X)$ ("ఏ" means "is subsemigroup of") then $S \cap \mathscr{K}(X)$ (where $\mathscr{K}(X)$ is the set of transformations whose range has cardinality one: the constant functions) will be abbreviated to $\mathscr{K}(S)$. An element of $\mathscr{K}(X)$ with range $a$ will be written $\kappa_{a}$, and, further

$$
K(S)=\left\{x \in X: \kappa_{x} \in S\right\} .
$$

The following result is fundamental: in spirit, it goes back to Malcev (1952).

THEOREM 1.1. If $S, T \leqq \mathscr{T}(X)$ with $\mathscr{K}(S), \mathscr{K}(T) \neq \square$ and $\phi: S \rightarrow T$ is an isomorphism then

$$
\mathscr{K}(S) \phi=\mathscr{K}(T)
$$

and there exists a bijection $g: K(S) \rightarrow K(T)$ such that

for all $a \in K(T), \alpha \in S$.

$$
a \alpha \phi=a g^{-1} \alpha g
$$

PROOF. Let $\kappa_{a} \in \mathscr{K}(T)$, and $\alpha \in S$ be such that $\alpha \phi=\kappa_{a}$. Choose $\kappa_{b} \in \mathscr{K}(S)$. Then $\kappa_{b} \alpha=\kappa_{b \alpha} \in S$ and $\left(\kappa_{b \alpha}\right) \phi=\kappa_{b} \phi \alpha \phi=\kappa_{b} \phi \kappa_{a}=\kappa_{a}$. Hence $\mathscr{K}(S) \phi \geqq \mathscr{K}(T)$ and by the dual result for $\phi^{-1}$ we have $\mathscr{K}(S) \phi=\mathscr{K}(T)$.

We define $g: K(S) \rightarrow K(T)$ by putting $a g=b$ when $\kappa_{a} \phi=\kappa_{b}$. Clearly $g$ is bijective. Note that for $a \in K(T), a g^{-1} \in K(S)$. Since $K(S) \alpha \subseteq K(S)$ for all $\alpha \in S$, we have $a g^{-1} \alpha \in K(S)$ so that $a g^{-1} \alpha g$ is well defined. 
Moreover for $\alpha \in S$,

$$
\kappa_{a g} \alpha \phi=\left(\kappa_{a} \alpha\right) \phi=\left(\kappa_{a \alpha}\right) \phi=\kappa_{a \alpha g}=\kappa_{a g} g^{-1} \alpha g \text {. }
$$

Hence $a g \alpha \phi=a g g^{-1} \alpha g$ and since $g$ is onto $K(S)$ we have the result.

If $S \leqq \mathscr{T}(X)$, let Aut $S$ be the group of automorphism of $S$ and Inn $S$ the set of automorphisms of $S$ of the form $\alpha \rightarrow g^{-1} \alpha g ; \alpha \in S$, for $g \in \mathscr{G}(X)$.

Further, if $Y \subseteq X$ let $\operatorname{Inn}_{Y} S$ be those inner automorphisms of $S$ induced by $\mathscr{G}(X, Y)$ and put

$$
\text { Aut }_{Y} S=\{\phi \in \text { Aut } S ; y \alpha \phi=y \alpha \text { for all } \alpha \in S, y \in Y\}
$$

The following result reduces the calculation of Aut $S$ to that of Aut $_{Y} S$ (for certain semigroups).

THEOREM 1.2. If $S \leqq \mathscr{T}(X)$ and $g^{-1} S g \subseteq S$ for all $g \in \mathscr{G}(X, K(S))$ where $K(S) \neq \square$ then

$$
\text { Aut } S=\operatorname{Aut}_{K(S)} S \operatorname{Inn}_{K(S)} S \text {. }
$$

Proof. Let $\phi \in$ Aut $S$. As in (1.1) we have

$$
a \alpha \phi=a g^{-1} \alpha g ; a \in K(S), \alpha \in S,
$$

where $g$ is a permutation of $K(S)$. Extend $g$ to a permutation of $X$ so that $g \in \mathscr{G}(X, K(S))$, let $\psi_{g}$ denote the automorphism of $S, \alpha \rightarrow g^{-1} \alpha g$ and put $\zeta=\phi \psi_{g^{-1}}$. Then $\zeta \in$ Aut $S$ and $\phi=\zeta \psi_{g}$. We show $\zeta \in$ Aut $_{K(S)} S$. Let $a \in K(S), \alpha \in S$. We then have

$$
\begin{aligned}
a(\alpha \zeta) & =\alpha\left(\alpha \phi \psi_{g^{-1}}\right) \\
& =a g \alpha \phi g^{-1} \\
& =a g\left(g^{-1} \alpha g\right) g^{-1}(\text { Since } a g \in K(S)) \\
& =a \alpha .
\end{aligned}
$$

If $S=\mathscr{T}(X, Y)$ then it is easy to see that $S$ satisfies the hypotheses of (1.2) with $K(S)=Y$. Hence we have a

Corollary. Aut $\mathscr{T}(X, Y)=\operatorname{Aut}_{Y} \mathscr{T}(X, Y) \operatorname{Inn}_{Y} \mathscr{T}(X, Y)$.

We observe that $\operatorname{Inn}_{Y} \mathscr{T}(X, Y)$ consists of all the mappings $g^{-1} \cdot g$, $g \in \mathscr{G}(X, Y)$.

The following result sets out some simple properties of $\operatorname{Aut}_{K(S)} S$.

LEMMA 1.3. If $S \leqq \mathscr{T}(X)$ and $\phi \in \operatorname{Aut}_{K(S)} S$ then

(i) $\phi^{-1} \in \operatorname{Aut}_{K(S)} S$

(ii) If $X \alpha \subseteq K(S)$ then $(\alpha \beta) \phi=\alpha \phi \beta .(\alpha, \beta \in S)$

(iii) If $\kappa \in \mathscr{K}(S)$ then $\kappa \phi=\kappa$. 
THEOREM 1.4. If $\mathscr{T}(X) \geqq S \geqq \mathscr{T}(X, Y)$ then $X \alpha=X \alpha \phi$, for all $\alpha \in \mathscr{T}(X, Y)$, $\phi \in \operatorname{Aut}_{K(S)} S$.

Proof. For $\alpha \in \mathscr{T}(X, Y)$ we construct $\varepsilon_{X \alpha}$ as follows

$$
\begin{aligned}
x \varepsilon_{X \alpha} & =x \text { when } x \in X \alpha \\
& =c \text { for some } c \in X \alpha, \text { otherwise. }
\end{aligned}
$$

We note that $\varepsilon_{X z} \in S$ and $x \varepsilon_{X \alpha}=x$ if and only if $x \in X \alpha$. Since $X \alpha \subseteq K(S)$ and $\alpha \varepsilon_{X \alpha}=\alpha$ we have

$$
\alpha \phi=\left(\alpha \varepsilon_{X \alpha}\right) \phi=\alpha \phi \varepsilon_{X \alpha}, \text { by (1.3). }
$$

It follows that $X \alpha \phi \subseteq X \alpha$.

Conversely, if we define $\varepsilon_{X \alpha \phi}$ analogously to $\varepsilon_{X \alpha}$ we have by (1.3), parts (i) and (ii),

$$
\alpha=\alpha \phi \phi^{-1}=\left(\alpha \phi \varepsilon_{X \alpha \phi}\right) \phi^{-1}=\alpha \phi \phi^{-1} \varepsilon_{X \alpha \phi}=\alpha \varepsilon_{X \alpha \phi} .
$$

Thus $X \alpha \subseteq X \alpha \phi$ and the result follows.

\section{Automorphisms of $\mathscr{I}(X, 2)$}

In this section we shall calculate the automorphism of $\mathscr{T}(X, Y)$ when $|Y|=2$. In $\$ 3$ we shall use these results to calculate $\mathscr{T}(X, Y)$ for arbitrary $Y$.

Hence, for our present purposes, we identify $Y$ with the set $2=\{0,1\}$ and abbreviate $\mathscr{T}(X, 2)$ to $T$. Moreover for $\alpha \in T$ we shall write $\alpha=\xi_{A}$ where $A=1 \alpha^{-1}$ and $\xi_{A}$ is the characteristic function of $A$ (i.e. $x \xi_{A}=1, x \in A ; 0$ otherwise).

LEMMA 2.1. If $A, B \subseteq X$ then

$$
\begin{aligned}
\xi_{A} \xi_{B} & =\xi_{A} \text { when } 1 \in B, 0 \notin B, \\
& =\xi_{A} \text {, when } 1 \notin B, 0 \in B, \\
& =\xi_{\square} \text { when } 1 \notin B, 0 \notin B, \\
& =\xi_{X} \text { when } 1 \in B, 0 \in B .
\end{aligned}
$$

(Note: when it is unambiguous we shall write $A^{\prime}$ for $X \backslash A$ ).

We make two

Definitions. (i) If $\phi \in$ Aut $T$ we define $\pi: \mathscr{P}(X) \rightarrow \mathscr{P}(X)$ by $A \pi=B$, when $\xi_{A} \phi=\xi_{B}$. Clearly $\pi$ is a permutation of $\mathscr{P}(X)$.

(ii) Let $Z \subseteq X$. A permutation of $\mathscr{P}(X), \pi$, will be called $Z$-admissible on $\mathscr{P}(X)$ if for all $A \in \mathscr{P}(X)$,

(1) $X \pi=X, \square \pi=\square$ 
(2) $A^{\prime} \pi=A \pi^{\prime}$

(3) $Z \cap A=Z \cap A \pi$.

It will be instructive to construct some $Z$-admissible permutations. Firstly, call a partition on $\mathscr{P}(X)$ into two subsets, $\mathscr{P}_{1}$ and $\mathscr{P}_{2}$, decisive if, for each $A \in \mathscr{P}(X)$, precisely one of $A$ and $A^{\prime}$ belong to $\mathscr{P}_{1}$ (equally, $\mathscr{P}_{2}$ ). titions.

The following device (due to R. P. Sullivan) yields a supply of decisive par-

Let $a \in X$. Define $\mathscr{P}_{1}$ by demanding -

$$
A \in \mathscr{P}_{1} \text { if and only if } a \in A
$$

— and put $\mathscr{P}_{2}=\mathscr{P}(X) \mid \mathscr{P}_{1}$. Then $\mathscr{P}_{1}, \mathscr{P}_{2}$ is the desired partition.

Now take $Z \subseteq X$, put $W=X \mid Z$, and let $\mathscr{P}_{1}, \mathscr{P}_{2}$ be a decisive partition of $\mathscr{P}(W)$, with $\square \in \mathscr{P}_{1}$. The mapping $\mathscr{P}_{1} \rightarrow \mathscr{P}_{2}$ given by $A \rightarrow A^{\prime} ; A \in \mathscr{P}_{1}$, is a bijection, so that $\left|\mathscr{P}_{1}\right|-\left|\mathscr{P}_{2}\right|$. It follows that if $\mathscr{M}_{1}, \mathscr{M}_{2}$ is another decisive partition then $\left|\mathscr{P}_{1}\right|=\left|\mathscr{M}_{1}\right|$. Let $\square \in \mathscr{M}_{1}$ and $\pi: \mathscr{P}_{1} \rightarrow \mathscr{M}_{1}$ be any bijection such that $\square \pi=\square$. Extend $\pi$ to $\mathscr{P}(W)$ by defining, for $A \in \mathscr{P}_{1}, A^{\prime} \pi=(A \pi)^{\prime}$. The construction is completed by writing, for each $A \in \mathscr{P}(X)$.

$$
A \pi=(A \backslash Z) \pi \cup(A \cap Z),
$$

where the $\pi$ on the right is that of the previous sen tence.

Then $\pi$ is $Z$-admissible on $\mathscr{P}(X)$.

The following two results indicate the connections between Definitions (i) and (ii).

Lemma 2.2. If $\phi \in \mathrm{Aut}_{2} T$ and $\pi$ is defined as in definition (i) then $\pi$ is 2-admissible.

Proof. We show $\pi$ has properties (1), (2) and (3) of Definition (ii). Now $\kappa_{0}=\xi_{\square}$ and $\kappa_{1}=\xi_{X}$. Since $\phi \in$ Aut $_{2} T$ property (1) follows easily from (1.3) (iii). Let $A, B \subseteq X$ and put $\xi_{A} \xi_{B}=\xi_{C}$. By (1.3) (ii) $\xi_{A} \phi \xi_{B}=\zeta_{C} \phi$ so that $\xi_{A \pi} \xi_{B}=\xi_{C \pi}$. If we take $B$ such that $1 \notin B, 0 \in B$ we have, by (2.1), $\xi_{C}=\xi_{A} \xi_{B}=\xi_{A^{\prime}}$, so that $\xi_{C_{\pi}}=\xi_{A^{\prime} \pi^{\prime}}$. On the other hand $\xi_{A \pi} \xi_{B_{\pi}}=\xi_{A^{\prime} \pi}$ so $\xi_{A^{\prime} \pi}=\xi_{A \pi^{\prime}}$, and the second property follows.

To see (3) we note that $\alpha|2=\alpha \phi| 2$. Hence $x \xi_{A}=x \xi_{A}$ for $x=0,1$; and all $A \in \mathscr{P}(X)$. It suffices to observe that $x \xi_{A}=0$ or 1 according as $x \notin A$ or $x \in A$.

We also have the converse:

LeMmA 2.3. If $\pi$ is 2-admissible on $\mathscr{P}(X)$ and

$$
\xi_{A} \phi=\xi_{A \pi} \text { for all } A \subseteq X,
$$

then $\phi \in \mathrm{Aut}_{2} T$. 
Proof. With $\phi$ as in the statement of the Lemma, it is clear that $\phi$ is bijective - since $\pi$ is a permutation of $\mathscr{P}(X)$.

It only remains to demonstrate that

$$
\xi_{A} \phi \xi_{B} \phi=\left(\xi_{A} \xi_{B}\right) \phi \text { and } \xi_{A} \phi\left|2=\xi_{A}\right| 2 \text {; for all } A, B \subseteq X .
$$

The second equatily is clear from (3). Then $\xi_{A} \phi \xi_{B} \phi=\xi_{A} \phi \xi_{B}$ so we show

$$
\left(\xi_{A} \xi_{B}\right) \phi=\xi_{A} \phi \xi_{B} ; A, B \subseteq X .
$$

Now if $1 \notin B, 0 \notin B$ or if $1 \in B, 0 \in B$ then property (1) and an application of (2.1) give the result. If $1 \in B, 0 \notin B$ then $\xi_{A} \xi_{B}=\xi_{A}$ by the same Lemma and

$$
\left(\xi_{A} \xi_{B}\right) \phi=\xi_{A} \phi=\xi_{A} \phi \xi_{B} .
$$

Finally if $1 \notin B, 0 \in B$ then $\xi_{A} \xi_{B}=\xi_{A} ; A \subseteq X$, (again by (2.1)) and $\left(\xi_{A} \xi_{B}\right) \phi$ $=\left(\xi_{A^{\prime}}\right) \phi=\xi_{A^{\prime} \pi}=\xi_{A \pi^{\prime}}$, by property (2). Hence $\left(\xi_{A} \xi_{B}\right) \phi=\xi_{A \pi^{\prime}}=\xi_{A \pi} \xi_{B}=\xi_{A} \phi \xi_{B}$. Finally if $1 \notin B, 0 \in B$ then $\xi_{A} \xi_{B}=\xi_{A^{\prime}} ; A \subseteq X$, (again by (2.1)) and $\left(\xi_{A} \xi_{B}\right) \phi=\left(\xi_{A^{\prime}}\right) \phi$ $=\xi_{A^{\prime} \pi}=\xi_{A \pi^{\prime}}$, by property (2). Hence $\left(\xi_{A} \xi_{B}\right) \phi=\xi_{A \pi^{\prime}}=\xi_{A \pi} \xi_{B}=\xi_{A} \phi \xi_{B}$.

Denote the set of automorphisms of $T$ induced by 2-admissible permutations of $\mathscr{P}(X)$ by Tap $T$. From the previous two Lemmas and the Corollary to $(1.2)$ we have

$$
\text { Aut } T=\operatorname{Tap} T \operatorname{Inn}_{2} T
$$

Let $\tau$ be the 2-cycle on $X$ which permutes 0 and 1 . All elements of $\mathscr{G}(X, 2)$ are of the form $g \tau^{n} ; n=1$ or 2 where $g \mid 2=1_{2}$ (the identity of $\mathscr{T}(2)$ ). It follows from (*) that Aut $T$ is just the mappings of the form

$$
\xi_{A} \phi=\tau^{n} g^{-1} \xi_{A \pi} g \tau^{n} \text { where } g \mid 2=\mathbf{1}_{2} .
$$

Now $g^{-1} \xi_{A \pi} g=\xi_{A \pi g} g=\xi_{A \pi g}$ and it is straightforward to show that $A \rightarrow A \pi g$ is 2-admissible when $g \mid 2=\mathfrak{l}_{2}$. Hence all automorphisms have the form

$$
\xi_{A} \phi=\tau^{n} \xi_{A \pi} \tau^{n} ; n=0 \text { or } 1,
$$

where $\pi$ is 2 -admissible. Hence each $\phi \in$ Aut $T$ may be written as a product

$$
\phi=p t^{n}(n=0,1)
$$

where $p \in \operatorname{Tap} T$ and $t=\tau \cdot \tau \in$ Aut $T$.

THEOREM 2.5. Aut $T \cong Z_{2} \times_{H} \Pi_{2}(X)$, the semi-direct product of the group of integers modulo 2 and the group of 2-admissible permutations of $\mathscr{P}(X)$, with connectiog homomorphism $H: Z_{2} \rightarrow \operatorname{Aut} \Pi_{2}(X)$ given by

$$
\pi(n H)=\tau^{n} \pi \tau^{n} .
$$

Proof. Let $D$ be the group consisting of the identity automorphsim and $t$. By the above

$$
\text { Aut } T=\operatorname{Tap} T \cdot D=D \cdot \operatorname{Tap} T .
$$


Now for all $A \subseteq X$,

$$
\xi_{A} t=\tau \xi_{A} \tau=\xi_{A \tau} \tau=\xi_{(A \tau)^{\prime}} .
$$

Hence $t \notin \operatorname{Tap} T$ since $A \rightarrow(A \tau)^{\prime}$ is not 2-admissible (consider $(2 \tau)^{\prime}$ ), so that $D \cap$ Tap $T$ is trivial. From $\left({ }^{*}\right)$, if $p \in \operatorname{Tap} T$ then $p t=p^{\prime}$ or $t p^{\prime}$, for some $p^{\prime} \in \operatorname{Tap} T$. Since $t \notin \operatorname{Tap} T$ we may exclude the former. Hence $t p t=t^{2} p^{\prime}=p^{\prime} \in \operatorname{Tap} T$. It follows that Tap $T$ is normal in Aut $T$.

From these remarks we may conclude Aut $T$ is a semi-direct product of $D$ and Tap $T$ and the map

$$
t^{n} p \rightarrow(n, \pi)
$$

(where $p \in \operatorname{Tap} T$ is induced by $\pi$ ) is bijective and induces a group operation on $Z_{2} \times \Pi_{2}(X)$. Moreover

$$
t^{n_{1}} p_{1} t^{n_{2}} p_{2}=t^{n_{1}+n_{2}} t^{n_{2}} p_{1} t^{n_{2}} p_{2},
$$

where $n_{1}, n_{2} \in Z_{2}, p_{1}, p_{2} \in \operatorname{Tap} T$. If $p_{1}$ and $p_{2}$ are induced by $\pi_{1}$ and $\pi_{2}$ respectively, then it is easy to see that $t^{n_{2}} p_{1} t^{n_{2}} \in \operatorname{Tap} T$ is induced by $\tau^{n_{2}} \pi \tau^{n_{2}}$. It follows that

$$
\left(n_{1}, \pi_{1}\right) \cdot\left(n_{2}, \pi_{2}\right)=\left(n_{1}+n_{2}, \tau^{n_{2}} \pi_{1} \tau^{n_{2}} \pi_{2}\right)=\left(n_{1}+n_{2}, \pi_{1}\left(n_{2} H\right) \pi_{2}\right) . \|
$$

Note. It is easy to see that the inner automorphisms of $T$ have the form $\xi_{A} \rightarrow \tau^{n} \xi_{A g} \tau^{n}$ where $g \in \mathscr{G}(X), g \mid 2=\mathfrak{\imath}_{2}$. It follows that Aut $T>\operatorname{Inn} T$ when we may find a 2-admissible permutation not of the form $A \rightarrow A g, g$ as above. This is the case when $|X|>2$. For example, take $X=\{0,1, x\}$ and let $\pi$ be the mapping

$$
\begin{gathered}
\{0\} \rightarrow\{0, x\} \rightarrow\{0\},\{1\} \rightarrow\{1, x\} \rightarrow\{1\} . \\
\square \rightarrow \square, X \rightarrow X,\{0,1\} \rightarrow\{0,1\},\{x\} \rightarrow\{x\} .
\end{gathered}
$$

Then $\pi$ is 2 -admissible - but it does not preserve cardinality.

\section{Automorphisms of $\mathscr{I}(X, Y),|Y| \geqq 3$}

As in Clifford and Preston (1957), (Vol. 2, page 241), we shall use the notation

$$
\alpha=\left(\begin{array}{l}
X_{i} \\
y_{i}
\end{array}\right)
$$

to mean that $\alpha \in \mathscr{T}(X)$ has range $\left\{y_{i} ; i \in I\right\}$ and $X_{i}=y_{i} \alpha^{-1}$.

In this section we shall consider the automorphisms of $\mathscr{T}(X, Y)$ for $|Y| \geqq 3$. Choose a subset of $Y$ containing precisely two elements 0 and 1 , and denote it by 2 .

The following theorem enables us to determine the action of automorphisms on $\mathscr{T}(X, 2)$. 
THEOREM 3.1. If $\phi \in \mathrm{Aut}_{Y} \mathscr{T}(X, Y)$ then

$$
\phi \mid \mathscr{T}(X, 2) \in \mathrm{Aut}_{2} \mathscr{T}(X, 2)
$$

Proof. By (1.4) $\phi \mid \mathscr{T}(X, 2): \mathscr{T}(X, 2) \rightarrow \mathscr{T}(X, 2)$. If $\alpha \in \mathscr{T}(X, 2)$ then $\alpha \phi^{-1} \in \mathscr{T}(X, 2)$, by (1.3) and another application of (1.4); and since $\alpha=\alpha \phi^{-1} \phi$ we have that $\phi \mid \mathscr{T}(X, 2)$ is onto.

Now it is clear that $\phi \mid \mathscr{T}(X, 2)$ is a monomorphism so it remains only to show that

$$
y \alpha \phi=y \alpha ; \text { for all } y \in 2, \alpha \in \mathscr{T}(X, 2) .
$$

But $2 \subseteq Y$ and the above formula follows from the definition of $\left.A_{4} \mathscr{T}\right) X, Y$ ). $\|$

As in $\$ 2$ we may regard $\mathscr{T}(X, 2)$ as consisting of characteristic functions, $\xi_{A}, A \subseteq X$. By the above result and Lemma (2.2) we have $\xi_{A} \phi=\xi_{A \pi}$ where $\pi: \mathscr{P}(X) \rightarrow \mathscr{P}(X)$ is 2-admissible.

THEOREM 3.2. If $\phi \in \operatorname{Aut}_{Y} \mathscr{T}(X, Y)$ and 2 is any doubleton subset of $Y$ then

$$
\left(\begin{array}{c}
X_{i} \\
y_{i}
\end{array}\right) \phi=\left(\begin{array}{c}
X_{i} \pi \\
y_{i}
\end{array}\right) ;\left(\begin{array}{c}
X_{i} \\
y_{i}
\end{array}\right) \in \mathscr{T}(X, Y)
$$

where $\pi$ is 2-admissible.

Proof. Let $\alpha \in \mathscr{T}(X, Y), \alpha=\left(\begin{array}{l}X_{i} \\ y_{i}\end{array}\right)$. If $A \subseteq X$ it is easily seen that $\alpha \xi_{A}$ $=\xi_{A \alpha^{-1}}$. Hence $\xi_{A(\alpha \phi)^{-1}}=\alpha \phi \xi_{A}=\left(\alpha \xi_{A}\right) \phi=\left(\xi_{A \alpha^{-1}}\right) \phi=\xi_{A x^{-1} \pi}$. It follows that $A(\alpha \phi)^{-1}=A \alpha^{-1} \pi$ for all $A \subseteq X, \alpha \in \mathscr{T}(X, Y)$. Choosing, for fixed $i \in I$, $A=\left\{y_{i}\right\}$ we have

$$
A \alpha^{-1} \pi=X_{i} \pi=y_{i}(\alpha \phi)^{-1}
$$

and since $X \alpha=X \alpha \phi$ (by (1.4)) the result follows. $\|$

COROLlary.

$$
\left(\begin{array}{c}
X_{i} \\
y_{i}
\end{array}\right) \phi^{-1}=\left(\begin{array}{c}
X_{i} \pi^{-1} \\
y_{i}
\end{array}\right) \text {. }
$$

Lemma 3.3. If $\phi \in \operatorname{Aut}_{Y} \mathscr{T}(X, Y)$ and $A, B \subseteq X$ with $A \cap B=\square$ then $A \pi \cap B \pi=\square$ and

$$
(A \cup B) \pi=A \pi \cup B \pi
$$

Proof. Since $|Y| \geqq 3$ we may choose $\{0,1, t\} \subseteq Y$. Put $\alpha=\left(\begin{array}{lll}A & B & C \\ 0 & 1 & t\end{array}\right)$ where $C=X \mid(A \cup B)$. Clearly $\alpha \in \mathscr{T}(X, Y)$. We define $\varepsilon \in \mathscr{T}(X, Y)$ as follows

$$
\begin{aligned}
& x \varepsilon=0 ; x \in 2 \cup(X \backslash Y) \\
& x \varepsilon=x ; \text { otherwise }
\end{aligned}
$$

- then $\alpha \varepsilon=\left(\begin{array}{cc}A \cup B & C \\ 0 & t\end{array}\right)$. 
Moreover $(\alpha \varepsilon) \phi=\alpha \phi \varepsilon$ and so

$$
\left(\begin{array}{cc}
(A \cup B) \pi & C \pi \\
0 & t
\end{array}\right)=\left(\begin{array}{ccc}
A \pi & B \pi & C \pi \\
0 & 1 & t
\end{array}\right) \varepsilon=\left(\begin{array}{cc}
A \pi \cup B \pi & C \pi \\
0 & t
\end{array}\right) .
$$

LEMMA 3.4. If $a \in X$ then $|\{a\} \pi|=1$.

Proof. Let $a \in X$ and take $d \in\{a\} \pi$. Choose $C \subseteq X, C \neq \square$, such that $C \pi=\{b\} \subseteq\{a\} \pi$. Now $\pi^{-1}$ corresponds to the automorphism $\phi^{-1}$, and it follows readily from (3.3) that $\pi^{-1}$ preserves set containment. Hence $C \pi \pi^{-1}$ $\subseteq\{a\} \pi \pi^{-1}$, so that $C=\{a\}$.

Define $g: X \rightarrow X$ by putting $x g=y$ when $\{x\} \pi=\{y\}$. Since $\pi$ is a permutation of $\mathscr{P}(X), g$ is one to one.

LEMMA 3.5. $\pi$ is $Y$-admissible.

Proof. Clearly we need only prove that

$$
Y \cap A=Y \cap A \pi \text {. }
$$

Let $D$ be a subset of $Y,|D|=2$, and let $\sigma$ be the $D$-admissible permutation of $X$ corresponding to $D$. If $\alpha \in \mathscr{T}(X, Y), \alpha=\left(\begin{array}{c}X_{i} \\ y_{i}\end{array}\right)$, then

$$
\alpha \phi=\left(\begin{array}{c}
X_{i} \pi \\
y_{i}
\end{array}\right)=\left(\begin{array}{c}
X_{i} \sigma \\
y_{i}
\end{array}\right)
$$

It follows that $\pi=\sigma$ so we have

$$
D \cap A \pi=D \cap A \text { for all } D \subseteq Y \text { with }|D|=2,
$$

and the result is now immediate.

By putting $A=\{y\}$ (where $y \in Y$ ) in (*) we obtain

COROLLARY. $y g=y$ for all $y \in T$. $\|$

The job is finished by

Lemma 3.6. $A \pi=A g(=\{a g ; a \in A)\}) ; A \subseteq X$.

Proof. Let $A \subseteq X$. If $a \in A$ then

$$
A \pi=((A \mid a) \cup\{a\}) \pi=(A \mid a) \pi \cup\{a\} \pi, \text { by (3.3). }
$$

Since $\{a\} \pi=\{a g\}, A g \subseteq A \pi$.

On the other hand if $x \in A \pi$ then $A \pi=\{x\} \cup A \pi \backslash\{x\}$. Since $\pi$ is onto we may take $\{x\}=B \pi, A \pi \backslash\{x\}=0 \pi$ where $B$ and $C$ are non-empty disjoint subsets of $X$. From the preceding result $B \pi=\{x\} \supseteq B g$ and since $g$ is one to one $B=\{b\}$ where $b g=x$. Moreover, 


$$
A=A \pi \pi^{-1}=(B \pi \cup C \pi) \pi^{-1}=B \cup C
$$

since $B \pi \cap C \pi=\square$. Hence $A \supseteq B=\{b\}$ and $x=b g$ where $b \in A$. $\|$

We may now deduce that $g \in \mathscr{G}(X, Y)$. For, by the above result, $X=X \pi$ $=X g$, so that $g$ is onto, and by the corollary to (3.5) $g \mid Y=\iota_{Y} \in \mathscr{G}(Y)$.

The main result of this paper is

THEOREM 3.7. If $|Y| \geqq 3$ then

$$
\text { Aut } \mathscr{T}(X, Y)=\left\{g^{-1} \cdot g: g \in \mathscr{G}(X, Y)\right\} \text {. }
$$

Proof. If $\phi \in \operatorname{Aut}_{Y} \mathscr{T}(X, Y)$ and $\alpha \in \mathscr{T}(X, Y), \alpha=\left(\begin{array}{c}X_{i} \\ y_{i}\end{array}\right)$ then by the foregoing $\alpha \phi=\left(\begin{array}{c}X_{t} g \\ y_{i}\end{array}\right)=g^{-1}\left(\begin{array}{c}X_{i} \\ y_{i}\end{array}\right)=g^{-1} \alpha g$ (since $y g=y ; y \in Y$ ). Hence Aut $_{Y} \mathscr{T}(X, Y) \subseteq \operatorname{Inn}_{Y} \mathscr{T}(X, Y)$ and (1.2) gives the result.

Corollary. If $|Y| \geqq 3$,

$$
\operatorname{Aut} \mathscr{T}(X, Y) \cong \mathscr{G}(X, Y)
$$

\section{Extensions}

These results may be extended slightly to include (a class of) transformation semigroups which contain $\mathscr{T}(X, Y),|Y|>2$.

If $S$ is a transformation semigroup then we shall call $S Y$-complete if $\mathscr{T}(X)$ $\geqq S \geqq \mathscr{T}(X, Y)$ and $K(S)=Y$.

If $S$ is such a semigroup, then $\mathscr{T}(X, Y)$ is a left ideal of $S$. By (1.4) and (1.3)(i)

$$
\operatorname{Aut}_{Y} S \mid \mathscr{T}(X, Y) \subseteq \operatorname{Aut}_{Y} \mathscr{T}(X, Y)
$$

and by (3.7) any automorphism of $\mathscr{T}(X, Y)$ is inner, induced by some $g \in \mathscr{G}(X, Y)$. Hence if $\phi \in$ Aut $_{Y} S, \beta \in S$, and $\alpha \in \mathscr{T}(X, Y)$ we have

$$
(\beta \alpha) \phi=g^{-1} \beta \alpha g
$$

where $g \in \mathscr{G}(X, Y)$. Hence

$$
(\beta \phi)(\alpha \phi)=(\beta \alpha) \phi=g^{-1} \beta \alpha g=g^{-1} \beta g g^{-1} \alpha g g=g^{-1} \beta g(\alpha \phi) .
$$

It follows that

$$
\beta \phi \alpha=g^{-1} \beta g \alpha \text { for all } \alpha \in \mathscr{T}(X, Y)
$$

Since we may separate any two elements of $X$ by mappings in $\mathscr{T}(X, Y)$ we have $\beta \phi=g^{-1} \beta g$. We have proved

THEOREM 4.1. If $S$ is $Y$-complete with $Y \mid>2$ then $\operatorname{Aut}_{Y} S=\operatorname{Inn}_{Y} S$.

By (1.2) we have 
Corollary. If $S$ is as above and $g^{-1} S g \subseteq S$ for all $g \in \mathscr{G}(X, Y)$ then

$$
\text { Aut } S=\operatorname{Inn}_{Y} S \cong \mathscr{G}(X, Y) . \|
$$

Magill (1966) has studied the semigroup

$$
\mathscr{S}(X, Y)=\{\alpha \in \mathscr{T}(X) ; Y \alpha \subseteq Y\} .
$$

It is easy to see that $S \leqq \mathscr{T}(X)$ is $Y$-complete if and only if $\mathscr{P}(X, Y) \geqq S$ $\geqq \mathscr{T}(X, Y)$.

Since $g^{-1} \mathscr{S}(X, Y) g \subseteq \mathscr{S}(X, Y)$ for all $g \in \mathscr{G}(X, Y)$ we have from the corollary to (4.1)

THEOREM. 4.2 If $|Y|>2$ then

$$
\text { Aut } \mathscr{S}(X, Y)=\operatorname{Inn}_{Y} \mathscr{S}(X, Y) \cong \mathscr{G}(X, Y)
$$

\section{Isomorphisms of $\mathscr{I}(X, Y)$}

Let $X^{1}$ and $X^{2}$ be sets with $Y^{m} \subseteq X^{m}$ for $m=1$ and 2 . Denote $\mathscr{T}\left(X^{m}, Y^{m}\right)$ by $T^{m}$. The result of this section is

THEOREM. 5.1 If $T^{1} \cong T^{2}$ then $\left|Y^{1}\right|=\left|Y^{2}\right|$. Moreover,

if $\left|Y^{1}\right|=\left|Y^{2}\right|=1$ then $T^{1} \cong T^{2}$

if $\left|Y^{1}\right|=\left|Y^{2}\right|=2$ then $T^{1} \cong T^{2}$ if and only if $2^{\left|X^{1}\right| Y^{1} \mid}=2^{\left|X^{2}\right| Y^{2} \mid}$ and

if $\left|Y^{1}\right|=\left|Y^{2}\right|>2$ then $T^{1} \cong T^{2}$ if and only if $\left|X^{1}\right| Y^{1}|=| X^{2}\left|Y^{2}\right|$.

PROOF. The first assertion is a consequence of (1.1) while the second is trivial.

To see sufficiency in the final case, let $g_{1}$ and $g_{2}$ be bijections,

$$
g_{1}: Y^{1} \rightarrow Y^{2} \text { and } g_{2}: X^{1} \mid Y^{1} \rightarrow X^{2} \backslash Y^{2} \text {. }
$$

Define $g: X^{1} \rightarrow X^{2}$ by demanding that $g \mid Y^{1}=g_{1}$ and $g\left|X^{1}\right| Y^{1}=g_{2}$. Clearly $\alpha \rightarrow g^{-1} \alpha g: \alpha \in T^{1}$ is the required isomorphism. Note we have made no use of $\left|Y^{m}\right|>2$.

Now assume $2^{\left|X^{1}\right| Y^{1} \mid}=2^{\left|X^{2}\right| Y^{2} \mid}$ and set $\left.Y^{m}=0^{m}, 1^{m}\right\}$ for $m=1$ and 2 . We may immediately dismiss the possibility $\left|X^{m}\right|<\infty$ for then $\left|X^{1}\right| Y^{1}|=| X^{2}\left|Y^{2}\right|$ and we are in the case discussed above: henceforth we assume that $\left|X^{m}\right|$. and hence $\left.\left|X^{m}\right| Y^{m} \mid\right)$ is infinite.

Form decisive partitions of $\mathscr{P}\left(X^{m} \backslash Y^{m}\right): \mathscr{P}_{1}^{m}, \mathscr{P}_{2}^{m}$ for $m=1$ and 2. Now $\left|\mathscr{P}\left(X^{m} \mid Y^{m}\right)\right|=\left|\mathscr{P}_{1}^{m}\right|+\left|\mathscr{P}_{1}^{m}\right|=\max \left(\left|\mathscr{P}_{1}^{m}\right|,\left|\mathscr{P}_{2}^{m}\right|\right)=\left|\mathscr{P}_{1}^{m}\right| .\left(\right.$ Since $\left|\mathscr{P}_{1}^{m}\right|=\left|\mathscr{P}_{2}^{m}\right|$.) It follows that $\left|\mathscr{P}_{1}^{1}\right|=\left|\mathscr{P}_{1}^{2}\right|$ and we may choose a bijection, $\pi: \mathscr{P}_{1}^{1} \rightarrow \mathscr{P}_{1}^{2}$. The The construction is now a slight extension of one used in $\S 2$. Assume $\pi$ fixes $\square$. 
One extends $\pi$ to a bijection from $\mathscr{P}\left(X^{1}\right)$ to $\mathscr{P}\left(X^{2}\right)$ with the properties
(1) $\square \pi=\square$
(2) $\left(X^{1} \backslash A\right) \pi=X^{2} \backslash A \pi$
(3) $0^{1} \in A$ if and only if $0^{2} \in A \pi$ and $1^{1} \in A$ if and only if $1^{2} \in A \pi$.

It is now possible to prove that $\xi_{A} \in T^{1} \rightarrow \xi_{A \pi} \in T^{2}$ is an isomorphism by the methods of Lemma (2.3).

This completes the proof of sufficiency in the third assertion of the theorem. To see necessity it suffices to note that $\left|T^{m}\right|=2^{\left|X^{m}\right|}$ for $m=1,2$.

It only remains to prove necessity in the final case. Let $\phi: T^{1} \rightarrow T^{2}$ be an isomorphism with $\left|Y^{m}\right|>2$. By $\S 3$, Aut $_{Y^{m}} T^{m}$ consists of mappings of the form $h^{-1} \cdot h$ where $h \in \mathscr{G}\left(X^{m}\right)$ and $h \mid Y^{m}=\imath_{Y m}$. Hence Aut $y_{Y^{m}} T^{m} \cong \mathscr{G}\left(X^{m} \mid Y^{m}\right)$, so that it is sufficient to show $A_{Y^{2}} T \cong A_{Y^{2}} T^{2}$ to have $\left|X^{1}\right| Y^{1}|=| X^{2}\left|Y^{2}\right|$. (By a well known property of the symmetric group: See Scott (1964).)

The required isomorphism is

$$
\zeta \rightarrow \phi^{-1} \zeta \phi: \quad \zeta \in \operatorname{Aut}_{Y^{1}} T^{1} .
$$

This mapping is clearly a monomorphism so we need only show

$$
\phi^{-1} \text { Aut }_{Y} T^{1} \phi=\text { Aut }_{Y^{2}} T^{2},
$$

Let $y \in Y^{2}, \alpha \in T^{2}$, and $\zeta \in$ Aut $_{Y^{1}} T^{1}$. Taking $\zeta$ in the form indicated above and using the expression for $y \beta \phi, \beta \in T^{1}$, obtained in (1.1), it is easy to see that

$$
y\left(\alpha \phi^{-1} \zeta \phi\right)=y \alpha .
$$

Hence $\phi^{-1}$ Aut $_{Y^{1}} T^{1} \phi \subseteq$ Aut $_{Y^{2}} T^{2}$ and the dual result for $\phi^{-1}: T^{2} \rightarrow T^{1}$ completes the proof. $\|$

Note. Whether $2^{A}=2^{B}$ implies $A=B ; A, B$ infinite cardinals, is an unsolved problem. It is true under the assumption of the Generalized Continuum Hypothesis. See Sierpinski (1958), page 155.

Many classes of transformation semigroups are determined by the cardinality of the underlying sets. For example two full symmetric groups are isomorphic if and only if the sets for which they are defined have the same cardinality. Theorem (5.1) shows, however, that $\mathscr{T}(X, Y)$ is not determined by the cardinals $|X|$ and $|Y|$. As an example, let $\mathbf{N}$ be the set of natural numbers and $\mathbf{N}_{1}\left(\mathbf{N}_{2}\right)$ be those natural numbers greater than $1(2)$. Then $\left|\mathbf{N}_{1}\right|=\left|\mathbf{N}_{2}\right|=\mathbf{N}_{0}$. However, $|\mathbf{N}| \mathbf{N}_{1} \mid$ $\neq|\mathbf{N}| \mathbf{N}_{2} \mid$ so that $\mathscr{T}\left(\mathbf{N}, \mathbf{N}_{1}\right)$ is not isomorphic to $\mathscr{T}\left(\mathbf{N}, \mathbf{N}_{2}\right)$. 


\section{References}

A. H, Clifford and G. B. Preston (1961, 1967), The algebraic theory of semigroups, Vols. I and II (Math. Surveys of the American Math. Soc., 7, 1961 and 1967).

K. D. Magill, Jr. (1966), 'Subsemigroups of S(X)', Math. Japon. 11, 109-115.

A. I. Malcev (1952), 'Symmetric groupoids', Mat. Sb. (N. S.) 13 (73), 136-151.

W. R. Scott (1964), Group Theory, (Prentice-Hall, 1964).

W. Sierpinski (1958), Cardinal and Ordinal Numbers, (Warszawa, 1958).

University of Western Australia

Nedlands

Western Australia 